\title{
A Unified Model for Hysteresis in Ferroic Materials
}

\author{
Ralph C. Smith*, Stefan Seelecke ${ }^{\dagger}$, Marcelo J. Dapino ${ }^{\ddagger}$, and Zoubeida Ounaies ${ }^{\S}$ \\ ${ }^{*}$ Center for Research in Scientific Computation, North Carolina State Univ., Raleigh, NC 27695 \\ ${ }^{\dagger}$ Mechanical and Aerospace Engineering, North Carolina State University, Raleigh, NC 27695 \\ ${ }^{\ddagger}$ Department of Mechanical Engineering, The Ohio State University, Columbus, OH 43210 \\ §Mechanical Engineering, Virginia Commonwealth University Richmond VA 23284-3015
}

\begin{abstract}
This paper provides a unified modeling framework for ferroelectric, ferromagnetic and ferroelastic materials operating in hysteretic and nonlinear regimes. Whereas the physical mechanisms which produce hysteresis and constitutive nonlinearities in these materials differ at the microscopic level, shared energy relations can be derived at the lattice, or mesoscopic, scale. This yields a class of models which are appropriate for homogeneous, single crystal compounds. Stochastic homogenization techniques are then employed to construct macroscopic models suitable for nonhomogeneous, polycrystalline compounds with variable effective fields or stresses. This unified methodology for quantifying hysteresis and constitutive nonlinearities for a broad class of ferroic compounds facilitates both material characterization and subsequent model-based control design. Attributes of the models are illustrated through comparison with piezoceramic, magnetostrictive and thin film SMA data.
\end{abstract}

Keywords: Unified free energy models, hysteresis, constitutive nonlinearities, ferroic materials

\section{Introduction}

Hysteresis and constitutive nonlinearities are an inherent property of ferroelectric, ferromagnetic and ferroelastic compounds under a wide range of operating frequencies and drive regimes as illustrated by quasistatic PZT5A, Terfenol-D and thin film SMA data in Figure 1. For certain applications, these nonlinear effects can be minimized through low drive operation or feedback mechanisms. In other cases, however, hysteresis and nonlinear dynamics are unavoidable and must be accommodated for accurate material characterization and subsequent model-based control design. For example, hysteresis inherent to the piezoceramic nanopositioning elements employed in all present atomic force microscope (AFM) designs can be mitigated at low frequencies through PID or robust feedback control laws $[4,5,13]$. However, at the high scan rates required for real-time product diagnostics or monitoring cellular processes, noise to signal ratios preclude the sole use of feedback control laws to mitigate hysteresis, and control designs utilizing model-based inverse compensators are under investigation [18]. Furthermore, for many applications utilizing SMA, maximal hysteresis is desired to optimize damping since energy dissipation is proportional to the area of the hysteresis loop. Hence, for civil applications in which SMA tendons are utilized to attenuate earthquake vibrations in buildings, operation in highly hysteretic pseudoelastic regimes is required. This necessitates the development of unified models which characterize hysteresis and constitutive nonlinearities in a manner which facilitates subsequent transducer design and model-based control implementation.

\footnotetext{
*Email: rsmith@eos.ncsu.edu; Telephone: 919-515-7552

${ }^{\dagger}$ Email: stefan_seelecke@ncsu.edu; Telephone: 919-515-5282

‡Email: dapino.1@osu.edu; Telephone: 614-688-3689

§Email: zounaies@vcu.edu; Telephone: 804-827-7025
} 


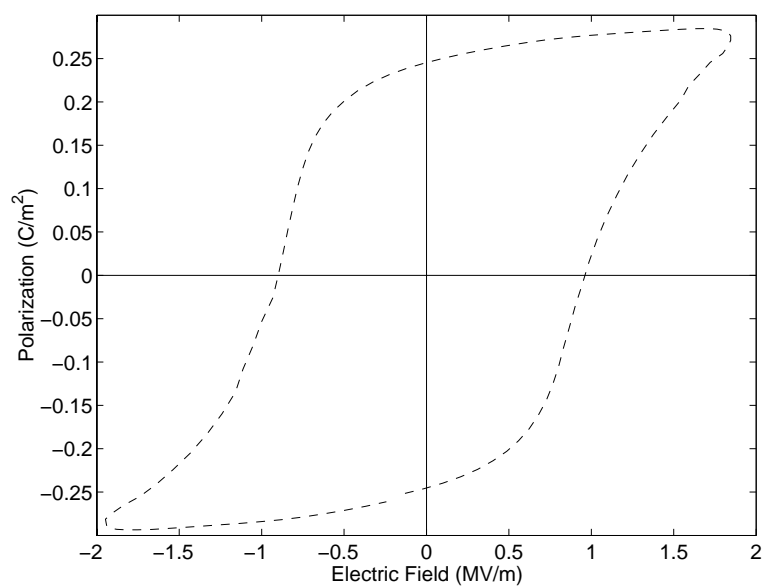

(a)

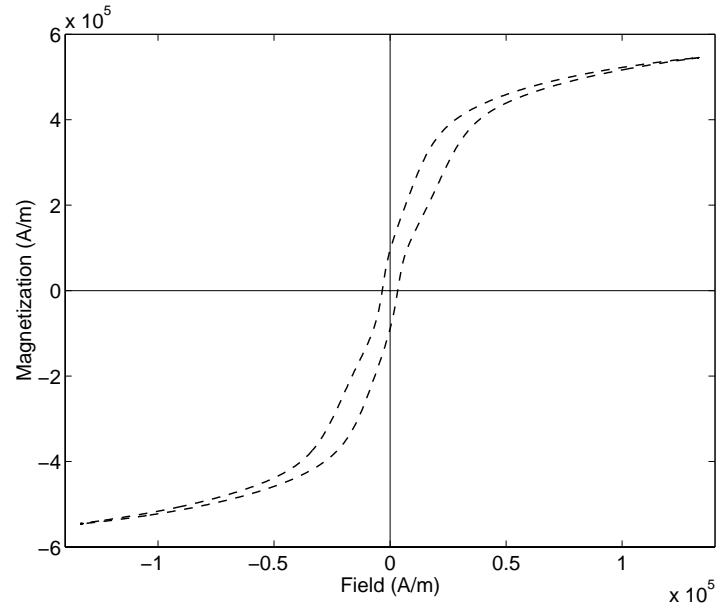

(b)

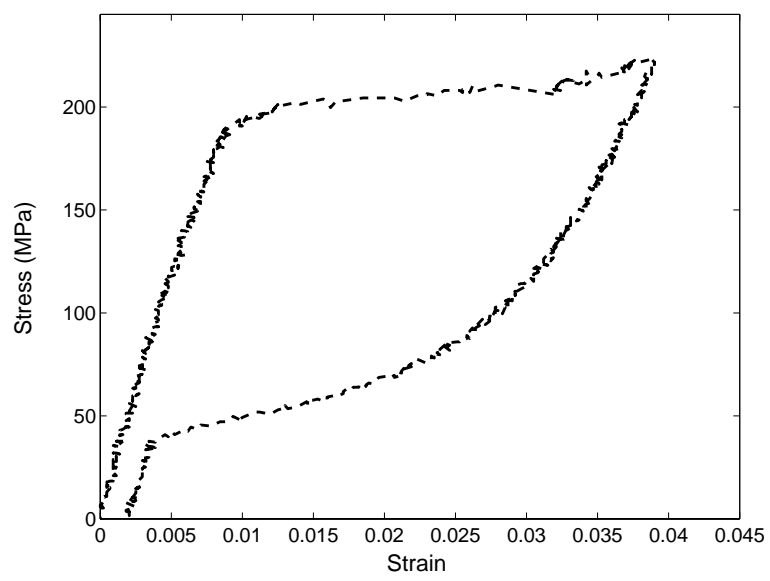

(c)

Figure 1. Hysteresis and constitutive nonlinearities exhibited by various ferroic compounds. (a) PZT5A data from [17], (b) Terfenol-D data from [6], and (c) thin-film SMA data from [8].

In this paper, we construct a hierarchy of unified models based on energy relations developed at the lattice level in combination with stochastic homogenization techniques to obtain macroscopic constitutive relations. In the first step of the development, Helmholtz and Gibbs energy relations are derived at the lattice level and combined with evolution laws to quantify the local average polarization, magnetization and strain in ferroelectric, ferromagnetic and ferroelastic compounds. These relations model hysteresis and nonlinear constitutive properties of homogeneous, single crystal compounds. The effects of material nonhomogeneities, polycrystallinity, and variable effective fields or stresses are subsequently incorporated through stochastic homogenization techniques to obtain macroscopic constitutive relations appropriate for bulk or thin film compounds. While developed in the context of piezoceramic, magnetostrictive and shape memory compounds, the methodology is sufficiently general to encompass a broad range of ferroic materials.

This methodology complements certain existing energy-based and phenomenological techniques used to develop unified hysteresis models for ferroic compounds. The models in [16] employ energy relations pertaining to the domain structure of ferroic compounds to characterize hysteresis and constitutive nonlinearities. A second unified approach for quantifying the hysteretic and nonlinear behavior of certain active materials was developed by Soukhojak and Chiang [22]. Based on phenomenological principles, this approach provides a rheological model quantifying the time and frequency-dependent behavior of a variety of ferroic compounds. Finally, Preisach mod- 
els have been employed to characterize hysteresis in a wide range of materials due their generality and broad mathematical basis $[1,2,3,7,9,12]$. The strengths and limitations of Preisach models for smart material applications, as well as an energy formulation for Preisach models based on the theory developed here, are summarized in [19].

Energy relations and local average polarization, magnetization and strain relations at the lattice level are developed in Section 2 and macroscopic models, developed through stochastic homogenization techniques, are presented in Section 3. Attributes of the unified models are illustrated through comparison with PZT5A, Terfenol-D and thin film SMA data in Section 4.

\section{Mesoscopic Models}

\subsection{Piezoceramic Materials}

As detailed in [20,21], consideration of statistical mechanics principles for fixed temperature regimes yields the piecewise quadratic Helmholtz relation

$$
\psi(P)= \begin{cases}\frac{1}{2} \eta\left(P+P_{R}\right)^{2} & , P \leq-P_{I} \\ \frac{1}{2} \eta\left(P-P_{R}\right)^{2} & , P \geq P_{I} \\ \frac{1}{2} \eta\left(P_{I}-P_{R}\right)\left(\frac{P^{2}}{P_{I}}-P_{R}\right) & ,|P|<P_{I}\end{cases}
$$

where $P_{I}$ and $P_{R}$ respectively denote the positive inflection point and polarization at which the minimum of $\psi$ occurs. From the potential energy relation $U_{E}=-\mathbf{p} \cdot \mathbf{E}$, for a dipole $\mathbf{p}$ in a field $\mathbf{E}$, it follows that an expression for the Gibbs energy is

$$
G=\psi-E P .
$$

The behavior of $G$ for an increasing field and the resulting local average polarization $\bar{P}$ are depicted in Figure 2 .

To characterize $\bar{P}$, it is necessary to quantify the fractions $x_{+}$and $x_{-}$of dipoles respectively having positive and negative orientations, and the likelihoods $p_{+-}$and $p_{-+}$of switching form a positive to negative orientation or vice versa. This is accomplished through consideration of the Boltzmann relation

$$
\mu(G)=C e^{-G V / k T}
$$

which quantifies the probability of achieving an energy level $G$ on a lattice volume $V$. The constant $C$ is chosen to guarantee integration to unity over all admissible inputs and $k$ denotes Boltzmann's constant.

By observing that the average polarization values due to positively and negatively oriented dipoles have the general formulations

$$
\left\langle P_{+}\right\rangle=\int_{P_{I}}^{\infty} P \mu(G) d P \quad, \quad\left\langle P_{-}\right\rangle=\int_{-\infty}^{-P_{I}} P \mu(G) d P,
$$

it follows that

$$
\left\langle P_{+}\right\rangle=\frac{\int_{P_{I}}^{\infty} P e^{-G(E, P, T) V / k T} d P}{\int_{P_{I}}^{\infty} e^{-G(E, P, T) V / k T} d P} \quad, \quad\left\langle P_{-}\right\rangle=\frac{\int_{-\infty}^{-P_{I}} P e^{-G(E, P) V / k T} d P}{\int_{-\infty}^{-P_{I}} e^{-G(E, P) V / k T} d P}
$$

It is illustrated in [21] that the use of the inflection point $P_{I}$, rather than the equilibrium value $P_{0}$, can be justified either from energy principles or asymptotic analysis. Similarly, the likelihoods $p_{+-}$and $p_{-+}$are given by

$$
\begin{aligned}
& p_{+-}=\sqrt{\frac{k T}{2 \pi m V^{2 / 3}}} \frac{e^{-G\left(E, P_{I}, T\right) V / k T}}{\int_{P_{I}}^{\infty} e^{-G(E, P, T) V / k T} d P} \\
& p_{-+}=\sqrt{\frac{k T}{2 \pi m V^{2 / 3}}} \frac{e^{-G\left(E,-P_{I}, T\right) V / k T}}{\int_{-\infty}^{-P_{I}} e^{-G(E, P, T) V / k T} d P}
\end{aligned}
$$



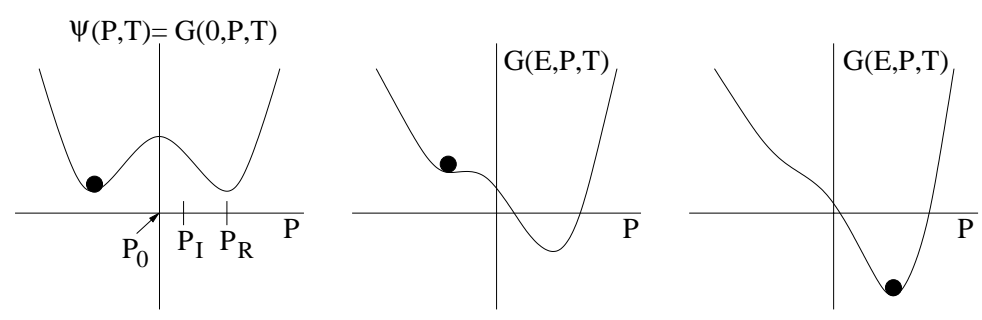

(a)
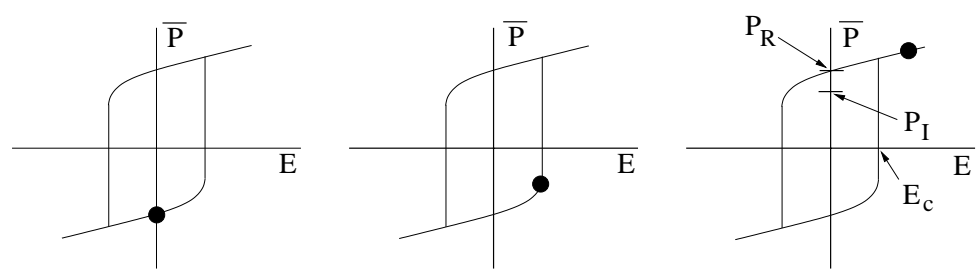

(b)

Figure 2. (a) Helmholtz energy $\psi$ and Gibbs energy $G$ for increasing field $E$. (b) Dependence of the local polarization $\bar{P}$ on the field $E$ at the lattice level.

where $m$ denotes the mass of the lattice volume $V$. The first term in the right hand side of each expression quantifies the frequency at which jumps are attempted.

The evolution of dipole fractions is quantified by the differential equations

$$
\begin{aligned}
& \dot{x}_{+}=-p_{+-} x_{+}+p_{-+} x_{-} \\
& \dot{x}_{-}=-p_{-+} x_{-}+p_{+-} x_{+}
\end{aligned}
$$

which can be simplified to

$$
\dot{x}_{+}=-p_{+-} x_{+}+p_{-+}\left(1-x_{+}\right)
$$

through the identity $x_{+}+x_{-}=1$. With the dipole fractions, transition likelihoods and average polarization values thus defined, the average local polarization at the lattice level is given by

$$
\bar{P}=x_{+}\left\langle P_{+}\right\rangle+x_{-}\left\langle P_{-}\right\rangle .
$$

It is demonstrated in [21] that for operating regimes in which relaxation effects are negligible, asymptotic analysis can be employed to reduce (9) to the algebraic relations

$$
\left[\bar{P}\left(E ; E_{c}, \xi\right)\right](t)= \begin{cases}{\left[\bar{P}\left(E ; E_{c}, \xi\right)\right](0)} & , \tau(t)=\emptyset \\ \frac{E}{\eta}-P_{R} & , \tau(t) \neq \emptyset \text { and } E(\max \tau(t))=-E_{c} \\ \frac{E}{\eta}+P_{R} & , \tau(t) \neq \emptyset \text { and } E(\max \tau(t))=E_{c}\end{cases}
$$

where

$$
\left[\bar{P}\left(E ; E_{c}, \xi\right)\right](0)= \begin{cases}\frac{E}{\eta}-P_{R} & , E(0) \leq-E_{c} \\ \xi & ,-E_{c}<E(0)<E_{c} \\ \frac{E}{\eta}+P_{R} & , E(0) \geq E_{c}\end{cases}
$$

denotes the initial dipole orientation and transition times are designated as

$$
\tau(t)=\left\{t \in\left(0, T_{f}\right] \mid E(t)=-E_{c} \text { or } E(t)=E_{c}\right\} .
$$

For single crystal materials which are isotropic, homogeneous, and exhibit uniform effective fields, the relations (9) or (10) hold throughout the compound and hence provide a macroscopic model quantifying the hysteretic 
and nonlinear relation between the input field and bulk polarization $P$. For general compounds, the stochastic homogenization techniques described in Section 3 must be combined with (9) or (10) to obtain macroscopic constitutive relations.

\subsection{Magnetostrictive Materials}

Under the assumption that magnetic moments have two preferred orientations, it is illustrated in [15] that an appropriate expression for the Helmholtz energy, for fixed temperature regimes, is

$$
\psi(M)= \begin{cases}\frac{1}{2} \eta\left(M+M_{R}\right)^{2} & , M \leq-M_{I} \\ \frac{1}{2} \eta\left(M-M_{R}\right)^{2} & , M \geq M_{I} \\ \frac{1}{2} \eta\left(M_{I}-M_{R}\right)\left(\frac{M^{2}}{M_{I}}-M_{R}\right) & ,|M|<M_{I}\end{cases}
$$

where $M_{I}$ and $M_{R}$ denote the inflection point and local remanence magnetization. Since the magnetostatic energy is given by $\mathcal{E}=\mu_{0} M H$, where $\mu_{0}$ is the magnetic permeability, the Gibbs energy can be expressed as

$$
G=\psi-H M
$$

by incorporating $\mu_{0}$ in $\psi$. Comparison of the ferromagnetic Helmholtz and Gibbs relations (11) and (12) with their ferroelectric analogues (1) and (2) reveals that they are identical if one equates $H$ with $E$ and $M$ with $P$.

The development of local average magnetization relations is analogous to that for ferroelectric materials and is detailed in [15]. For regimes in which thermally activated relaxation is significant, the local average magnetization is given by

$$
\bar{M}=x_{+}\left\langle M_{+}\right\rangle+x_{-}\left\langle M_{-}\right\rangle
$$

where $x_{+}$and $x_{-}$satisfy the differential equations (7) or (8), and $\left\langle M_{+}\right\rangle$and $\left\langle M_{-}\right\rangle$are given by

$$
\left\langle M_{+}\right\rangle=\frac{\int_{M_{I}}^{\infty} M e^{-G(H, M) V / k T} d M}{\int_{M_{I}}^{\infty} e^{-G(H, M) V / k T} d M} \quad, \quad\left\langle M_{-}\right\rangle=\frac{\int_{-\infty}^{-M_{I}} M e^{-G(H, M) V / k T} d M}{\int_{-\infty}^{-M_{I}} e^{-G(H, M) V / k T} d M} .
$$

For regimes in which thermally activated relaxation is negligible, asymptotic analysis yields the algebraic relation

$$
\left[\bar{M}\left(H ; H_{c}, \xi\right)\right](t)= \begin{cases}{\left[\bar{M}\left(H ; H_{c}, \xi\right)\right](0)} & , \tau(t)=\emptyset \\ \frac{H}{\eta}-M_{R} & , \tau(t) \neq \emptyset \text { and } H(\max \tau(t))=-H_{c} \\ \frac{H}{\eta}+M_{R} & , \tau(t) \neq \emptyset \text { and } H(\max \tau(t))=H_{c}\end{cases}
$$

where $\left[\bar{M}\left(H ; H_{c}, \xi\right)\right](0)$ denotes the initial moment orientation and transition points are specified by

$$
\tau(t)=\left\{t \in\left(0, T_{f}\right] \mid H(t)=-H_{c} \text { or } H(t)=H_{c}\right\} .
$$

Employing notation used for Preisach models [19], the initial moment orientation is specified by

$$
\left[\bar{M}\left(H ; H_{c}, \xi\right)\right](0)= \begin{cases}\frac{H}{\eta}-M_{R} & , H(0) \leq-H_{c} \\ \xi & ,-H_{c}<H(0)<H_{c} \\ \frac{H}{\eta}+M_{R} & , H(0) \geq H_{c}\end{cases}
$$

The local average relations (13) or (15) quantify the hysteretic relation between $H$ and $M$ for single crystal, homogeneous compounds, and they provide a kernel for the stochastic homogenization techniques in Section 3 for characterizing nonhomogeneous, polycrystalline materials. 


\subsection{Shape Memory Alloys}

The quasiplastic and pseudoelastic behavior inherent to shape memory compounds is due to phase transitions at the lattice, or mesoscopic, level between the high temperature austenite phase $A$ and low temperature martensite variants. In this discussion, we focus on applications, such as NiTi wires, characterized by uniaxial loads and hence we focus on the martensite twins $M^{+}$and $M^{-}$. Following the theory developed in [8, 11, 14], we treat a lattice volume $V$ as the fundamental element in the model. We subsequently let $x_{A}, x_{+}$and $x_{-}$ respectively denote the volume fractions of $A, M^{+}$and $M^{-}$layers in the SMA. The phase fractions necessarily satisfy the conservation relation

$$
x_{A}+x_{+}+x_{-}=1
$$

over all time.

To model the transition from stability of martensite variants at low temperatures to stability of the austenite phase at high temperatures, we consider the $C^{1}$ Helmholtz energy relation

$$
\psi(\varepsilon, T)= \begin{cases}\frac{E_{M}}{2}\left(\varepsilon+\varepsilon_{T}\right)^{2} & , \quad \varepsilon \leq-\varepsilon_{M}(T) \\ -\frac{E_{0}(T)}{2}\left(\varepsilon+\varepsilon_{0}(T)\right)^{2}+\psi_{0}(T) & , \quad-\varepsilon_{M}(T)<\varepsilon<-\varepsilon_{A}(T) \\ \frac{E_{A}}{2} \varepsilon^{2}+\Delta \beta(T) & , \quad|\varepsilon| \leq \varepsilon_{A}(T) \\ -\frac{E_{0}(T)}{2}\left(\varepsilon-\varepsilon_{0}(T)\right)^{2}+\psi_{0}(T) & , \quad \varepsilon_{A}(T)<\varepsilon<\varepsilon_{M}(T) \\ \frac{E_{M}}{2}\left(\varepsilon-\varepsilon_{T}\right)^{2} & , \quad \varepsilon \geq \varepsilon_{M}(T) .\end{cases}
$$

Here $\varepsilon$ denotes the shear strain in the layer, $\varepsilon_{M}$ is the stress-free equilibrium strain for martensite, and $E_{M}, E_{A}$ respectively denote the linear elastic moduli for martensite and austenite. The temperature-dependent inflection points $\varepsilon_{M}(T)$ and $\varepsilon_{A}(T)$ delineate the transition from concave parabolae, which represent unstable states, to convex regions, which represent stable austenite and martensite phase. The austenite minimum has the height $\Delta \beta(T)$ detailed in $[8]$.

To incorporate the effects of an applied stress $\sigma$, we consider the Gibbs energy relation

$$
G=\psi-\sigma \varepsilon
$$

As with the analogous ferroelectric Gibbs relation (2) and ferromagnetic relation (12), the inclusion of the work term has the effect of distorting the energy landscape in the direction of the applied force. In the absence of thermally activated relaxation, stable equilibria are computed by enforcing the necessary condition $\frac{\partial G}{\partial \varepsilon}=0$. To incorporate thermal activation, we consider the Boltzmann probability $\mu(G)$, given by $(3)$, which balances the elastic energy $G V$ over a reference volume $V$ with the thermal energy $k T$.

As detailed in $[8,14]$, the evolution of phase fractions, in the presence of thermal activation, is governed by the rate laws

$$
\begin{aligned}
& \dot{x}_{-}(t)=p_{A-} x_{A}(t)-p_{-} x_{-}(t) \\
& \dot{x}_{+}(t)=p_{A+} x_{A}(t)-p_{+} x_{+}(t) \\
& \dot{x}_{A}(t)=p_{-} x_{-}(t)-p_{A-} x_{A}(t)+p_{+} x_{+}(t)-p_{A+} x_{A}(t)
\end{aligned}
$$

which can be reduced to

$$
\begin{aligned}
& \dot{x}_{-}(t)=-\left(p_{-}+p_{A-}\right) x_{-}(t)-p_{A-} x_{+}(t)+p_{A-} \\
& \dot{x}_{+}(t)=-\left(p_{+}+p_{A+}\right) x_{+}(t)-p_{A+} x_{-}(t)+p_{A+}
\end{aligned}
$$

through the conservation relation (16). The likelihoods $p_{A \pm}$ that austenite will transform to $M^{ \pm}$, as well as the 
likelihoods $p_{ \pm}$that $M^{ \pm}$will transform either to austenite or the other martensite variant, are given by

$$
\begin{aligned}
& p_{A \pm}(\sigma, T)=\sqrt{\frac{k T}{2 \pi m V^{2 / 3}}} \frac{e^{-G\left( \pm \varepsilon_{A}, \sigma, T\right) V / k T}}{\int_{-\varepsilon_{A}}^{\varepsilon_{A}} e^{-G(\varepsilon, \sigma, T) V / k T} d \varepsilon} \\
& p_{ \pm}(\sigma, T)=\sqrt{\frac{k T}{2 \pi m V^{2 / 3}}} \frac{e^{-G\left( \pm \varepsilon_{M}, \sigma, T\right) V / k T}}{\int_{ \pm \varepsilon_{M}}^{ \pm \infty} e^{-G(\varepsilon, \sigma, T) V / k T} d \varepsilon}
\end{aligned}
$$

The evolution laws (19) and likelihoods (21) can be compared with their ferroelectric and ferromagnetic counterparts in (6) - (8).

To compute the local average strain for the reference volume, we make the assumption that thermal strains are small compared to mechanical strains and include only the latter component. As discussed in [8], this assumption is valid for bulk materials but may need to be modified for certain SMA thin film geometries. Under this assumption, the local average strains are given by

$$
\bar{\varepsilon}=\left\langle\varepsilon_{-}\right\rangle x_{-}+\left\langle\varepsilon_{+}\right\rangle x_{+}+\left\langle\varepsilon_{A}\right\rangle x_{A}
$$

where the expected strains due to $M^{ \pm}$and $A$ variants are given by

$$
\begin{aligned}
& \left\langle\varepsilon_{-}\right\rangle=\int_{-\infty}^{-\varepsilon_{M}} \varepsilon \mu(G(\varepsilon, \sigma, T)) d \varepsilon \\
& \left\langle\varepsilon_{+}\right\rangle=\int_{\varepsilon_{M}}^{\infty} \varepsilon \mu(G(\varepsilon, \sigma, T)) d \varepsilon \\
& \left\langle\varepsilon_{A}\right\rangle=\int_{-\varepsilon_{A}}^{\varepsilon_{A}} \varepsilon \mu(G(\varepsilon, \sigma, T)) d \varepsilon .
\end{aligned}
$$

The probability $\mu(G)$ of obtaining the energy $G$ is specified by the Boltzmann relation (3).

Because phase transitions in shape memory alloys can be driven by temperature, it is also necessary to quantify the temperature as a function of applied current $I$, convection to surrounding media, and heat transduction during phase transitions. As detailed in $[8,11,14]$, a balance of energy yields the differential equation

$$
\bar{c}(t) \dot{T}(t)=-h_{c} \Omega\left(T-T_{E}(t)\right)-\sum_{\alpha} h_{\alpha} \dot{x}_{\alpha}+J(t)
$$

where $\bar{c}(t)=\sum_{\alpha} c_{\alpha} x_{\alpha}(t)$ quantifies the average, phase-dependent specific heat. The first term on the right hand side quantifies the exchange of heat with surrounding media. Here $h_{c}, \Omega$ and $T_{E}$ respectively denote a convection coefficient, the SMA surface to volume ratio, and the temperature of the surrounding environment. The second right hand side term quantifies the latent heats of phase transitions where $h_{\alpha}$ denotes specific enthalpies (see $[8,14]$ for details regarding the formulation of $\left.h_{\alpha}\right)$. Finally, the expression

$$
J(t)=\bar{\rho}^{e}(t) \frac{I^{2}(t)}{\zeta^{2}},
$$

where $\bar{\rho}^{e}(t)$ and $\zeta^{2}$ respectively denote the average, phase-dependent electrical resistivity and SMA cross-sectional area, quantifies heat generation via Joule heating due to an applied current $I(t)$.

In combination, the coupled dynamic relations (22) and (24) characterize both stress driven and thermally activated strains at the lattice level in shape memory compounds. For homogeneous, single crystal compounds with constant effective stresses, these relations apply throughout the material and hence provide macroscopic relations quantifying the constitutive behavior of the material. The extensions required to characterize the constitutive nonlinearities and hysteresis inherent to nonhomogeneous, polycrystalline SMA compounds are addressed in the next section. 


\section{Macroscopic Models for Polycrystalline Compounds}

The ferroic models in Section 2 were derived at the lattice level and hence are mesoscopic in nature. For single crystal, homogeneous compounds, these relations hold uniformly throughout the material and hence can be used to model the macroscopic constitutive behavior of the materials. Stochastic homogenization techniques used to extend this theory to nonhomogeneous polycrystalline compounds with variable effective fields are summarized in this section.

We consider first the construction of macroscopic models for ferroelectric compounds. As detailed in [20, 21], variations in the lattice can be incorporated by assuming that local coercive fields $E_{c}$ are manifestations of an underlying lognormal distribution with density

$$
f\left(E_{c}\right)=c_{1} e^{-\left[\ln \left(E_{c} / \bar{E}_{c}\right) / 2 c\right]^{2}},
$$

to enforce the requirement that $E_{c} \geq 0$, whereas variable effective fields are assumed to be normally distributed about the applied field with the density

$$
\widetilde{f}\left(E_{e}\right)=c_{2} e^{-\left(E-E_{e}\right)^{2} / b} .
$$

The macroscopic polarization model combines the densities (26) and (27) to yield

$$
[P(E)](t)=C \int_{0}^{\infty} \int_{-\infty}^{\infty}\left[\bar{P}\left(E_{e}+E, E_{c}, \xi\right)\right](t) e^{-E_{e}^{2} / b} e^{-\left[\ln \left(E_{c} / \bar{E}_{c}\right) / 2 c\right]^{2}} d E_{e} d E_{c}
$$

where $\bar{P}$ is specified by (9) or (10) and $\xi$ denotes the initial distribution of dipoles. Details regarding the approximation of the integrals and implementation of the model are provided in [20,21].

The development of a macroscopic magnetization model follows in an analogous manner. In this case, we assume that magnetic coercive and effective fields can be characterized by the densities

$$
\begin{aligned}
& f\left(H_{c}\right)=c_{1} e^{-\left[\ln \left(H_{c} / \bar{H}_{c}\right) / 2 c\right]^{2}} \\
& \widetilde{f}\left(H_{e}\right)=c_{2} e^{-\left(H-H_{e}\right)^{2} / b} .
\end{aligned}
$$

Alternatively, one can employ a truncated normal distribution to characterize variations in $H_{c}$ as considered in [15]. The resulting macroscopic magnetization is quantified by the relation

$$
[M(H)](t)=C \int_{0}^{\infty} \int_{-\infty}^{\infty}\left[\bar{M}\left(H_{e}+H, H_{c}, \xi\right)\right](t) e^{-H_{e}^{2} / b} e^{-\left[\ln \left(H_{c} / \bar{H}_{c}\right) / 2 c\right]^{2}} d H_{e} d H_{c}
$$

where the kernel $\bar{M}$ is given by (13) or (15).

To extend the local average strain relation (22) to nonhomogeneous, polycrystalline ferroelastic compounds, we consider variations in the relative stress

$$
\delta(T)=\sigma_{A}(T)-\sigma_{M}(T)
$$

as well as variations in the effective stress $\sigma_{e}$. Under the assumption that $\delta$ and $\sigma_{e}$ are lognormally and normally distributed, this yields the macroscopic relation

$$
[\varepsilon(\sigma, T)](t)=C \int_{0}^{\infty} \int_{-\infty}^{\infty}\left[\bar{\varepsilon}\left(\sigma_{e}+\sigma, \delta, \xi\right)\right](t) e^{-\sigma_{e}^{2} / b} e^{-[\ln (\delta / \bar{\delta}) / 2 c]^{2}} d \sigma_{e} d \delta
$$

quantifying the strains due to input stresses and evolving temperatures.

A comparison of the ferroelectric model (28), ferromagnetic model (30) and ferroelastic model (32) reveals that all are comprised of coercive, relative and effective fields and stresses. The piecewise quadratic relations used to quantify the Helmholtz and Gibbs energies at the lattice level also yield highly analogous expressions

for the local average polarization $\bar{P}$, magnetization $\bar{M}$, and strain $\bar{\varepsilon}$ subsequently employed to construct the 
macroscopic relations (28), (30) and (32). In combination, this produces a unified modeling methodology for characterizing hysteresis and constitutive nonlinearities in a broad range of ferroelectric, ferromagnetic and ferroelastic compounds.

\section{Experimental Validation}

To illustrate the performance of the unified modeling methodology, we consider the characterization of hysteresis and constitutive nonlinearities in PZT5A, Terfenol-D, and thin film SMA. In all cases, quasistatic drive conditions were employed to minimize rate dependencies in the PZT and SMA and eddy current losses in the Terfenol-D.

\subsection{Experimental Validation for PZT5A}

To illustrate the performance and prediction capabilities of the ferroelectric model, we consider the characterization of hysteresis and constitutive nonlinearities for a rectangular $1.7 \mathrm{~cm} \times 0.635 \mathrm{~cm} \times 0.0381 \mathrm{~cm}$ PZT5A wafer. The data was collected at $0.2 \mathrm{~Hz}$ for an input field having a maximum value of $2.36 \times 10^{6} \mathrm{~V} / \mathrm{m}$. We note that this field corresponds to a maximum input voltage of $600 \mathrm{~V}$ with the field and voltage related by the approximate expression $E=V / t$ where $t=3.81 \times 10^{-4}$ m denotes the thickness of the wafer.

The parameters in the model were estimated through a least squares fit to the data yielding the values $P_{R}=0.04 \mathrm{C} / \mathrm{m}^{2}, \bar{E}_{c}=0.866010 \times 10^{6} \mathrm{~V} / \mathrm{m}, \eta=9.5 \times 10^{8}, c=0.4272 \mathrm{~V}^{2} / \mathrm{m}^{2}, b=1.9754 \times 10^{11} \mathrm{~V}^{2} / \mathrm{m}^{2}$ and $C=7.9926 \times 10^{-12}$. The model fit obtained with these values is compared with the experimental data in Figure 3. It is observed that the model accurately characterizes the observed hysteresis and saturation effects for this compound. Furthermore, with composite Gaussian quadrature routines used to approximate the integrals in (28), the algorithm is highly efficient to implement with full characterization requiring on the order of seconds on a workstation. Finally, examples illustrating the predictive capabilities of the model for PZT5H and PZT4 compounds as well as numerical examples illustrating that the model guarantees closure of biased minor loops can be found in [21].

\subsection{Experimental Validation for Terfenol-D}

To illustrate the performance of the magnetization model, we consider the characterization of the $H-M$ relation measured in a Terfenol-D transducer of the type depicted in Figure 4a. Details regarding the transducer

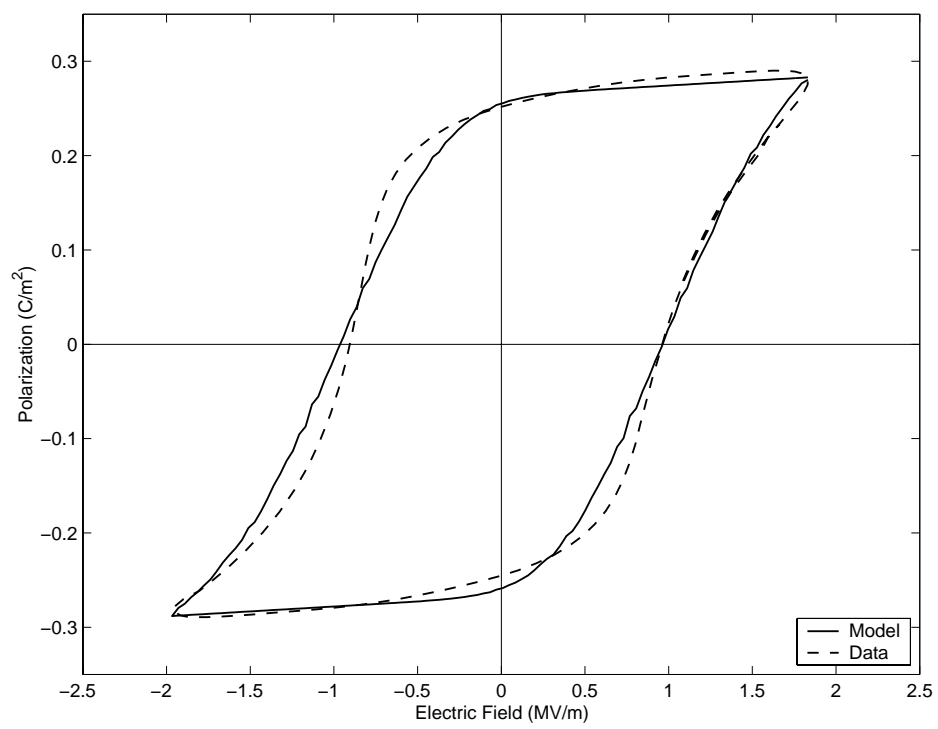

Figure 3. Model fit to $600 \mathrm{~V}$ data from a rectangular PZT5A wafer. 


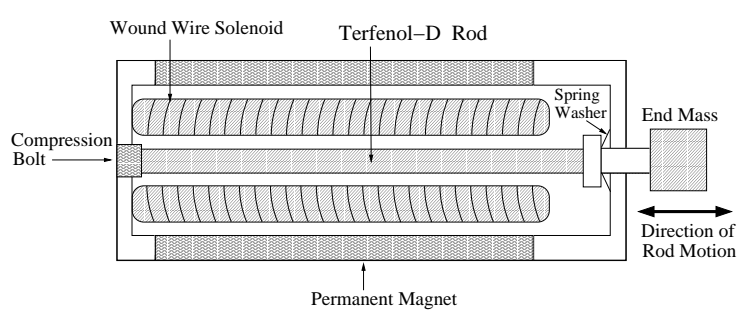

(a)

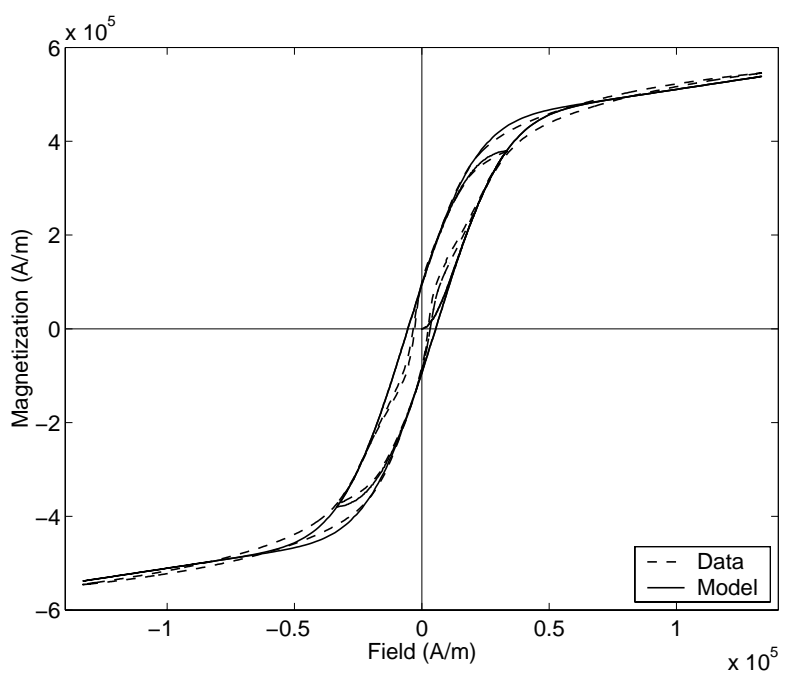

(b)

Figure 4. (a) Prototypical Terfenol-D transducer. (b) Experimental data (- - ) from [6] and model response $(-)$.

construction and manner through which the experimental data was collected are provided in [6]. We consider here data collected at $1 \mathrm{~Hz}$ with a prestress of $1 \mathrm{ksi}(6.9 \mathrm{MPa})$. Two input levels yields the moderate and high drive level magnetization data plotted in Figure 4b.

As detailed in [15], a least squares fit to the high drive level data was used to obtain the parameter values $M_{R}=3.7 \times 10^{4} \mathrm{~A} / \mathrm{m}, \eta=14, H_{c}=300 \mathrm{~A} / \mathrm{m}, b=1 \times 10^{8} \mathrm{~A}^{2} / \mathrm{m}^{2}, \bar{b}=8 \times 10^{8} \mathrm{~A}^{2} / \mathrm{m}^{2}, C=2.52 \times 10^{-8}$. We note that a truncated normal distribution was employed to model the coercive field distribution rather than the lognormal density (29). The resulting model fit at the high drive level and model prediction at the moderate drive level are plotted with the data in Figure 4b. It is observed that the model accurately quantifies the coercive, remanent, and saturation behavior of the $H-M$ relation. The primary discrepancy between the model prediction and data occurs at low field levels were the data exhibits a change in concavity which is typically attributed to crystalline anisotropies inherent to Terfenol-D. However, the general accuracy provided by the model is sufficient for material characterization, device design, and model-based control design.

\subsection{Experimental Validation for SMA}

The final example illustrates the characterization of a $8 \mu \mathrm{m}$ thick NiTi film constructed at the UCLA Active Materials Laboratory. Details regarding the experimental conditions, parameter estimation, and implementation of the model can be found in $[8,23]$.

The model (32) with the parameter values $E_{M}=14.0 \mathrm{GPa}, E_{A}=22.4 \mathrm{GPa}, \varepsilon_{T}=0.0233, \tau=1.7 \mathrm{~ms}$, $\rho=6450 \mathrm{~kg} \mathrm{~m}^{-3}, c_{V A}=c_{V M}=837.3 \mathrm{~J} \mathrm{~kg}^{-1} \mathrm{~K}^{-1}, \lambda_{A}=11.0 \times 10^{-6} \mathrm{~K}^{-1}$ and $\lambda_{M}=6.6 \times 10^{-6} \mathrm{~K}^{-1}$ is compared with quasistatic superelastic hysteresis data in Figure 5. It is observed that the model accurately quantifies both the leading curve and the asymmetric unloading curve as the stress is released. This provides an initial demonstration of the accuracy and efficiency of the model for characterizing SMA thin films.

\section{Concluding Remarks}

The theory presented here provides a unified methodology for quantifying hysteresis and constitutive nonlinearities inherent to a broad range of ferroic compounds including ferroelectric, ferromagnetic and ferroelastic materials. In the first step of the development, Helmholtz and Gibbs energy relations are combined with Boltzmann theory to provide lattice level, or mesoscopic, models which can be used to quantify hysteresis in homogeneous, single crystal compounds. Stochastic homogenization techniques are then employed to construct macroscopic 


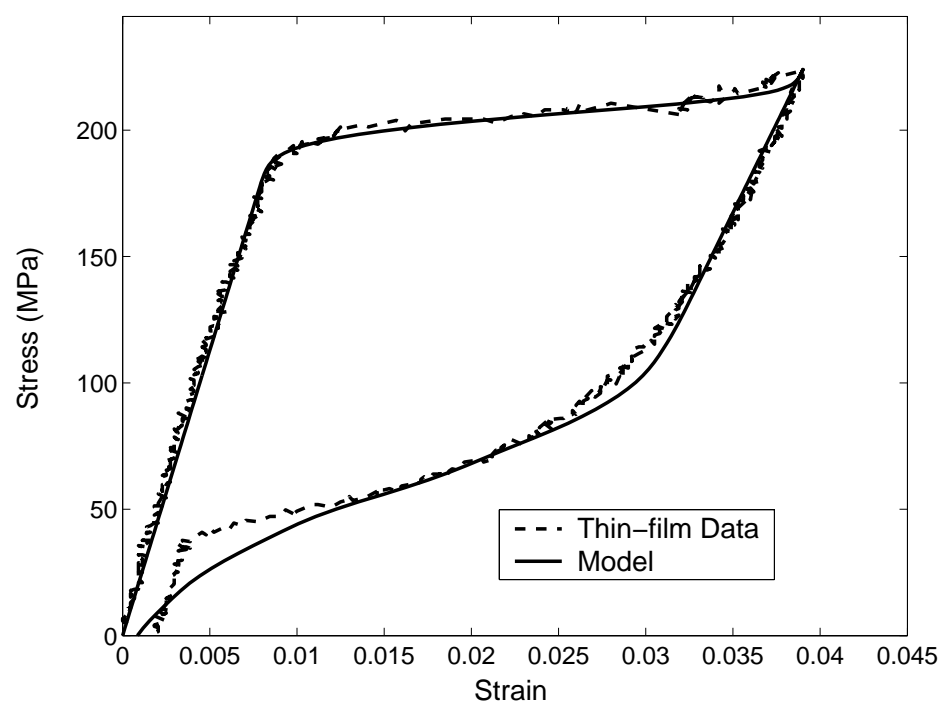

Figure 5. Experimental thin film NiTi data and model fit provided by (32).

models which incorporate the effects of polycrystallinity, material nonhomogeneities, and nonuniform effective fields.

Due to its generality, it is not surprising that the methodology bears certain similarities to Preisach models and it is illustrated in [19] that the free energy framework actually provides an energy basis for Preisach models. However, there are two important differences between the Preisach theory and that presented here. First, the free energy theory provides models having a small number of parameters, many of which can be estimated using physical properties of the data. This is in contrast to Preisach models which can have a large number of nonphysical parameters. Secondly, frequency and temperature effects are incorporated in the basis for the present model as compared with the necessity of utilizing vector-valued densities or parameters in Preisach models. Both properties augment the efficiency of the free energy framework for material characterization and subsequent model-based control design.

\section{Acknowledgements}

The authors thank Jason Woolman, UCLA, for providing the SMA hysteresis data used in this paper and Jordan Massad, North Carolina State University, for the SMA model simulations used to characterize this data. The research of R.C.S. was supported in part through the NSF grant CMS-009764 and in part by the Air Force Office of Scientific Research under the grant AFOSR-F49620-01-1-0107. The research of S.S. was supported in part by the National Science Foundation through the grant DMI-0134464. The research of M.J.D. was provided in part by The Ohio State University through startup funds.

\section{References}

Note: Center for Research in Scientific Computation Technical Reports can be accessed at the web site http://www.ncsu.edu/crsc/reports.html.

[1] A. Adly, I. Mayergoyz and A. Bergqvist, "Preisach modeling of magnetostrictive hysteresis," Journal of Applied Physics, 69(8), pp. 5777-5779, 1991.

[2] H.T. Banks, A.J. Kurdila G. and G. Webb, "Identification of hysteretic control influence operators representing smart actuators Part I: Formulation," Mathematical Problems in Engineering. 3, pp. 287-328, 1997. 
[3] M. Brokate and J. Sprekels, Hysteresis and Phase Transitions, Springer-Verlag, New York, 1996.

[4] M. Ashhab, M.V. Salapaka, M. Dahleh and I. Mezic, "Dynamical analysis and control of micro-cantilevers," Automatica, 1999.

[5] A. Daniele, S. Salapaka, M.V. Salapaka and M. Dahleh, "Piezoelectric scanners for atomic force microscopes: Design of lateral sensors, identification and control," Proc. of the ACC, San Diego, CA, 1999 pp. 253-257.

[6] M.J. Dapino, R.C. Smith, L.E. Faidley and A.B. Flatau, "A coupled structural-magnetic strain and stress model for magnetostrictive transducers," Journal of Intelligent Material Systems and Structures, 11(2), pp. 134-152, 2000.

[7] P. Ge and M. Jouaneh, "Modeling hysteresis in piezoceramic actuators," Precision Engineering, 17, pp. 211$221,1995$.

[8] J.E. Massad, R.C. Smith and G.P. Carman, "A free energy model for thin-film shape memory alloys," CRSC Technical Report CRSC-TR03-03; Proceedings of the SPIE, Smart Structures and Materials 2003, to appear.

[9] I.D. Mayergoyz, Mathematical Models of Hysteresis, Springer-Verlag, New York, 1991.

[10] J.M. Nealis and R.C. Smith, "Robust control of a magnetostrictive actuator," CRSC Technical Report CRSC-TR03-05; Proceedings of the SPIE, Smart Structures and Materials 2003, to appear.

[11] N. Papenfuß and S. Seelecke, "Simulation and control of SMA actuators," Proceedings of the SPIE, Smart Structures and Materials 1999, Volume 3667, pp. 586-595, 1999.

[12] J.B. Restorff, H.T. Savage, A.E. Clark and M. Wun-Fogle, "Preisach modeling of hysteresis in Terfenol-D," Journal of Applied Physics, 67(9), pp. 5016-5018, 1996.

[13] A. Sebastion and S. Salapaka, " $H_{\infty}$ loop shaping design for nano-positioning," 2002 American Control Conference, to appear.

[14] S. Seelecke and I. Müller, "Shape memory alloy actuators in smart structures - Modeling and simulation," ASME Applied Mechanics Reviews, to appear.

[15] R.C. Smith, M.J. Dapino and S. Seelecke, "A free energy model for hysteresis in magnetostrictive transducers," Journal of Applied Physics, 93(1), pp. 458-466, 2003.

[16] R.C. Smith and J.E. Massad, "A unified methodology for modeling hysteresis in ferroic materials," Proceedings of the 18th ASME Biennial Conference on Mechanical Vibration and Noise, 2001.

[17] R.C. Smith and Z. Ounaies, "A domain wall model for hysteresis in piezoelectric materials," Journal of Intelligent Material Systems and Structures, 11(1), pp. 62-79, 2000.

[18] R.C. Smith, M.V. Salapaka, A. Hatch, J. Smith and T. De, "Model development and inverse compensator design for high speed nanopositioning," Proc. 41st IEEE Conf. Dec. and Control, 2002, Las Vegas, NV.

[19] R.C. Smith and S. Seelecke, "An energy formulation for Preisach models," Proceedings of the SPIE, Smart Structures and Materials 2002, Volume 4693, pp. 173-182, 2002.

[20] R.C. Smith, S. Seelecke and Z. Ounaies, "A free energy model for piezoceramic materials," Proceedings of the SPIE, Smart Structures and Materials 2002, Volume 4693, pp. 183-190, 2002.

[21] R.C. Smith, S. Seelecke, Z. Ounaies and J. Smith, "A free energy model for hysteresis in ferroelectric materials," CRSC Technical Report CRSC-TR03-01; Journal of Intelligent Material Systems and Structures, submitted.

[22] A.N. Soukhojak and Y.-M. Chiang "Generalized rheology of active materials," Journal of Applied Physics, 88(11), pp. 6902-6909, 2000.

[23] J. Woolman, "Effect of atomic composition on the mechanical properties of thin film pseudoelastic nickel titanium," Master's Thesis, Mechanical and Aerospace Engineering Department, UCLA, in preparation. 